BMJ Open

Sport \&

Exercise

Medicine

\title{
Physical activity and sedentary time in a rural adult population in Malawi compared with an age-matched US urban population
}

Michael Pratt (D, ${ }^{1}$ James F Sallis, ${ }^{2}$ Kelli L Cain, ${ }^{2}$ Terry L Conway, ${ }^{2}$ Amparo PalaciosLopez, ${ }^{3}$ Alberto Zezza, ${ }^{3}$ Chad Spoon, ${ }^{2}$ Carrie M Geremia, ${ }^{2}$ Isis Gaddis, ${ }^{3}$ Akuffo Amankwah, ${ }^{3}$ Jed Friedman, ${ }^{3}$ Talip Kilic ${ }^{3}$

To cite: Pratt M, Sallis JF, Cain $\mathrm{KL}$, et al. Physical activity and sedentary time in a rural adult population in Malawi compared with an age-matched US urban population. BMJ Open Sport \& Exercise Medicine 2020;0: e000812. doi:10.1136/ bmjsem-2020-000812

Accepted 17 August 2020

\section{SLinked}

http://dx.doi.org/10.1136/ bmjsem-2020-000941

Check for updates

(C) Author(s) (or their employer(s)) 2020. Re-use permitted under CC BY-NC. No commercial re-use. See rights and permissions. Published by BMJ.

For numbered affiliations see end of article.

Correspondence to

Michael Pratt; mipratt@ucsd.ed

\section{ABSTRACT}

Objectives This study was designed to assess patterns of objectively measured physical activity (PA) and sedentary behaviour in a sample of adults in a rural setting from a lowincome Sub-Saharan African country (Malawi). The patterns of PA and sedentary behaviour in Malawi were compared with US data collected and analysed using the same methodology.

Methods The Malawi PA data were collected as part of a survey experiment on the measurement of agricultural labor conducted under the World Bank Living Standards Measurement Study program. ActiGraph accelerometers (model GT3X) were worn on the right hip in a householdbased sample of 414 working-age adults (15-85 years). Results Mean total and 95\% Cls for PA by category in min/ day for Malawi adults were: sedentary 387.6

(377.4-397.8), low-light 222.1 (214.7-229.5), high-light 136.3 (132.7-139.9), moderate 71.6 (68.8-74.5), vigorous $1.1(0.5-1.8)$ and moderate-to-vigorousphysical activity (MVPA) 72.8 (69.7-75.9). Mean of PA and sedentary behavior (min/day) summed across age and sex groups are compared between Malawi and US samples: sedentary behaviour, 387.6 vs 525.8 ( $p<0.001)$; low-light, 222.1 vs $217.0(p=n s)$; high-light, 136.3 vs $45.6(p<0.001)$; moderate, 71.6 vs 28.0 ( $p<0.001)$; vigorous, 1.1 vs 2.5 $(p<0.001)$; MVPA, 72.8 vs $30.5(p<0.001)$. Compared with the USA, Malawi participants averaged consistently less sedentary time/day and more minutes/day in all intensity levels of PA, except for low-light and vigorous PA.

Conclusion Overall, levels of MVPA and high-light activity in adults in Malawi were substantially higher and sedentary time was substantially lower than those observed in US samples using near identical data collection, scoring and analysis.

\section{INTRODUCTION}

Physical activity (PA) is an important contributor to global health, reducing the risk of morbidity and mortality from cardiovascular disease, cancer, diabetes and other non-communicable diseases (NCD). ${ }^{1}$ Over recent decades, physical inactivity (not meeting guidelines) and sedentary (sitting) time have become especially problematic for low-income and middle-income

\section{Summary box}

What is already known?

- Objectively measured daily time of MVPA in adults from upper-income and middle-income country urban samples from around the world ranges from approximately 30 to $50 \mathrm{~min}$.

- Several studies suggest that adults in rural Africa may be more active.

What are the new findings?

- Overall levels of MVPA and light PA are substantially higher in a sample of adults in rural Malawi (a lowincome African country) than have been observed in other population-based samples using hip-worn accelerometers.

- The prevalence of meeting the 150 min per week PA guideline is much higher in the Malawi sample (94\%) than the US sample (55\%).

- Sedentary time among adults in rural Malawi is correspondingly lower than seen in upper-income and middle-income country populations.

- Surprisingly, vigorous PA levels observed in adults in Malawi were actually lower than those for an agematched US urban sample, though levels were less than 3 min per day in both groups.

countries (LMIC) in which a majority of the preventable mortality from NCD occurs. ${ }^{2}$ Physical inactivity is estimated to cause more than five million deaths per year globally. ${ }^{3}$ Despite its importance, PA has rarely been a focus of public health efforts in LMIC. ${ }^{4}$ This may in part be due to limited population surveillance data on the prevalence of PA and sedentary time. ${ }^{5}$ While this data gap is closing, ${ }^{6}$ our understanding of patterns of PA and sedentary time in lower-income countries, rural areas and in Sub-Saharan Africa remains limited. ${ }^{7}$

As PA assessment methodology has evolved from self-report questionnaires to devicebased measures (eg, accelerometry) the gap 
between high-quality PA data in high-income versus lowincome countries is widening. Few studies with objective PA data have been published from rural populations of adults from low-income countries. The absence of this information hampers the ability to guide public health programmes and our understanding of the transition from rural to urban lifestyles. Urbanisation in LMICs is associated with increases in NCD and postulated decreases in PA and increases in sedentary time. ${ }^{2}$ Recent African data from population-based self-report surveys show substantial inactivity in many countries. ${ }^{6}$ A handful of studies with objective measures of PA suggest that PA, while higher than in high-income countries, is still much lower than optimal. ${ }^{8-11}$ The aim of the present study was to assess accelerometry-measured PA and sedentary time among adults (15+) that were active in agriculture and that were members of approximately 200 rural households that participated in a household survey that was implemented in Malawi, a low-income Sub-Saharan country. Use of standardised methodology from a multi-country study ${ }^{12}{ }^{13}$ allowed for comparison of patterns of PA in Malawi with those from age-matched US samples.

\section{METHODS}

\section{Malawi agricultural labour survey: design and purpose}

The Malawi PA data were collected as part of the 'Malawi Agricultural Labor Experiment 2016/17 which was conducted under the World Bank Living Standards Measurement Study (LSMS) program. The larger study collected agricultural labour data during the 2016/17 agricultural season in 20 rural enumeration areas (EA) spread evenly across the Ntcheu and Zomba districts. In each EA, 36 agricultural households were sampled at random and were randomly allocated to one of the three agricultural labour data collection designs - for a total of 240 households per design. All designs gathered self-reported information on the quantity (days and hours) of farming labour during the agricultural season - either through weekly visits, weekly phone interviews or a recall interview at the end of the season. To provide objective PA data for validity comparisons with the self-reported data on farm labour, each adult member of the households in the weekly visit arm was selected to wear accelerometers. The participation rate for the accelerometer component of the study was $86 \%$. Subjects were involved in the design, or conduct, or reporting, or dissemination plans of our research, providing feedback on survey design and protocol.

\section{US-based comparison studies}

Accelerometer data were collected in three US-based studies examining the relationship of the built environment to PA, sedentary behaviour and health. These studies were conducted in geographically and demographically diverse neighbourhoods in Seattle/King County, WA, and the Baltimore, MD-Washington, DC region. ${ }^{14}$ Participation rates were $26 \%$ (adults), $21 \%$ (seniors) and $36 \%$ (adolescents), which are generally consistent with other studies using mail/phone contact methods for recruitment. Detailed methods for these studies have been reported elsewhere. ${ }^{15-17}$ For present analyses, only participants within the age range of the Malawi sample (1585 years) were selected. These studies were approved by relevant Institutional Review Boards and required informed consent by adults and assent by adolescents.

\section{Accelerometer training for Malawi data collection}

A coordinating centre with extensive experience using accelerometers provided training and consultation. A train-the-trainer approach was used, whereby key members of the research team participated in a 4-day training in the USA, then replicated the accelerometer workshop to train local Malawian data collectors. The US coordinating centre provided ongoing online assistance during Malawian trainings. The train-the-trainer workshops included all topics required to implement data collection, including detailed instruction on accelerometer protocols, usage of a Microsoft Access database for participant tracking, and instruction and tips to improve wearing compliance. Trainings included consultation on managing concerns that were specific to implementing accelerometer measurement within the Malawian context.

\section{Accelerometer data collection and wearing protocol in Malawi}

ActiGraph accelerometers (model GT3X) were deployed to two working-age adults $(15+$ years $)$ in each sampled household, prioritising first those engaged in agriculture and then deploying accelerometers to additional working-age household members at random, as a function of the number of remaining accelerometers available for deployment. Accelerometers were distributed in person during a visit to each residence, and instructions for how to wear and care for the device were provided. Detailed procedures were implemented to ensure quality control during data collection. Participants were instructed to wear the accelerometer around the waist, with the meter positioned just above the right hipbone, using a belt that was provided during all waking hours (except when bathing or swimming) for 14 days. To encourage wearing the accelerometer, a log was provided for participants to record wear times, as well as sleep and wake times for each day. ${ }^{18}$ After 7 days of data collection, the local data collection team visited each participant to download and screen accelerometer data for device malfunction and wearing time. Data collectors also entered the daily logs into the Microsoft Access tracking database. The tracking database and accelerometer data files were transferred to the coordinating centre on a weekly basis for review. The coordinating centre screened all accelerometer data for valid wear time and provided feedback to the Malawi team. The goal was to collect data for at least 5 days per each 7-day wearing period. After 14 days, all data were downloaded and screened once again for wearing time and device malfunction. If at either time point 
a device malfunction was detected, a new accelerometer was deployed with instructions to wear for the number of days lost to equipment failure. Data collectors entered the logs and wearing information for the remaining days and transferred the tracking database and final accelerometer files to the coordinating centre. The coordinating centre completed the cleaning of the entries to prepare for data analysis.

\section{US studies}

Accelerometer data collection and wearing procedures were similar for the three US-based studies. ActiGraphs (model 7164) were deployed to one participant per household by mail for a 7-day wear period. Participants were instructed to wear the accelerometer around the waist above the right hipbone on a belt that was provided during all waking hours (except when bathing or swimming) and to complete a wearing log. If fewer than 5 wearing days were recorded, a second wearing of the device was requested for the number of days fewer than the requested 7 days.

\section{Common scoring protocol}

A standardised protocol for scoring accelerometer data was adapted from an international study on built environments and PA. ${ }^{12}{ }^{13}$ Data were aggregated to 60-s epochs, and the low-frequency extension (LFE) was activated in the GT3X devices used in Malawi. ${ }^{19}$ Data from the USA were collected with an older generation ActiGraph (7164) that did not have a filter option. The LFE was used with the GT3X devices to maximise comparability of activity estimates between samples in Malawi and the USA. Studies have shown that data collected with the 7164 are comparable to data collected with the newer generation devices (GT3X) when the LFE is applied. ${ }^{19} 20$

Data from the vertical axis were extracted from the triaxial devices (GT3X model) used in the Malawi study to match the vertical axis data collected with the uniaxial 7164 model in the US studies. Data from both countries were scored using Freedson adult cut points for moderate and vigorous PA (1952 counts/min for moderate and 5725 counts/min for vigorous or 'hard'; Freedson et al, 1998). ${ }^{21}$ Light activity was categorised as low-light (101-929 counts/min) or high-light (930-1951 counts/ min), and sedentary time was scored with the commonly used cut point of $\leq 100$ counts $/ \mathrm{min} .{ }^{22}{ }^{23} \mathrm{PA}$ and sedentary time were estimated as cumulative time spent in the different activity intensities over the course of a day. Nonwear time was defined as 60 or more consecutive zero counts, and wearing time was defined as all possible wearing time minus non-wear. Ten wearing hours were required for a valid day. MeterPlus version 5.0 was used for data scoring. Participants were included in the sample if they had one or more valid wearing days. Although the instructions were to remove the accelerometer prior to going to bed at night, some participants wore the monitor 24 hours a day, or kept the monitor under a pillow during sleeping, leading to enough non-zero counts for some sleeping time to be classified as wear time. To eliminate sleeping time from activity estimates, data recorded between 22:00 and 04:30 were eliminated prior to data scoring. For determining these universal times for sleep, we analysed the self-reported sleep and waking time from the wear logs and found that the time between 04:30 and 22:00 represented 95\% of the samples' self-reported awake times.

\section{Analysis plan and variables}

Average total minutes of activity accumulated in each intensity category per valid wearing day (excluding sleep period) were calculated for the entire sample and also stratified by age group and sex for each country. To compare PA and sedentary behaviour by country, age group, and sex, the General Linear Model (GLM) procedure in SPSS Version 26 was used. Main effects for country, age group, and sex, and the interaction effects for country*age group and country*sex were tested in the model for each activity-intensity category (sedentary, lowlight, high-light, moderate, vigorous, and MVPA) and total daily counts, controlling for accelerometer-wearing time and age as covariates in each model. The per cent of wearing time spent in each intensity category by sex and country was calculated using the marginal means from the GLM models and used for graphical comparisons (figure 1). To calculate the per cent of the sample meeting the current PA guidelines of $150 \mathrm{~min}$ or more per week of moderate-to-vigorous physical activity (MVPA), the average MVPA per valid day variable was multiplied by 7 to obtain an estimate of average weekly minutes of MVPA. Non-parametric Kruskal-Wallis tests with independent samples were used to compare the per cent meeting PA guidelines by country, age group and sex.

\section{RESULTS}

PA-intensity measures are presented separately for the $\mathrm{n}=414$ Malawi participants and the $\mathrm{n}=3258$ US participants. Because PA consistently varies by sex and age, findings are summarised separately for male and female participants and by 4 age groups: adolescents (15-19 years old), younger adults (20-39 years), middle-aged adults (40-64 years) and older adults (65-85 years old). As shown in table 1, average age of participants in the Malawi and US samples was 35.1 and 48.6 years old, respectively. Women comprised $56.0 \%$ of the Malawi sample and $50.3 \%$ of the US sample. On average, accelerometers were worn for 14.2 and 13.6 hours per day by Malawi and US participants. The number of days that participants wore accelerometers was 13.3 and 6.3 days in Malawi and the USA, respectively, with little variation across age groups.

\section{Physical activity and sedentary time by age groups and country}

Table 2 shows the average minutes per day for each of the scored activity-intensity levels: sedentary time, low-light 


\section{MALAWI, MALES}

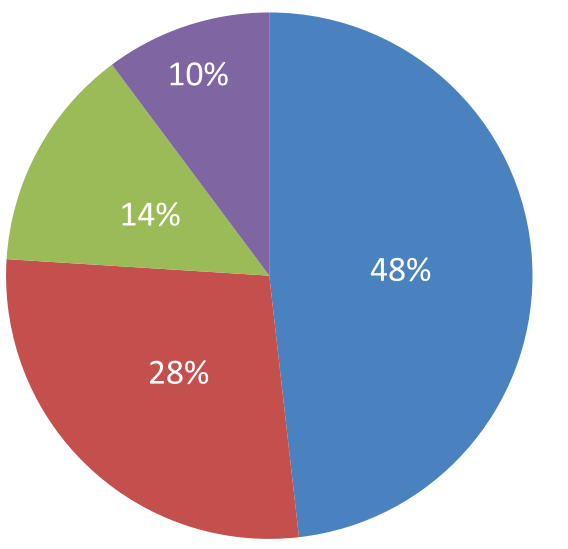

\section{US, MALES}

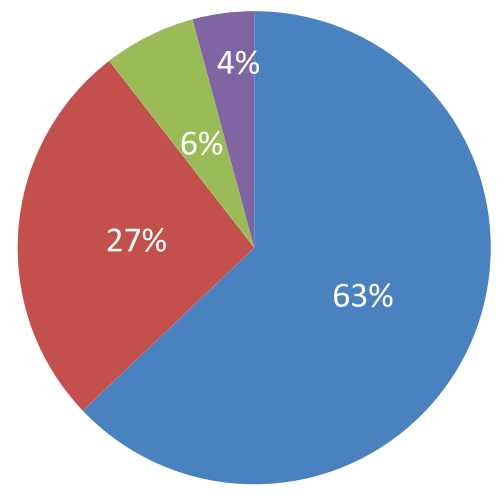

MALAWI, FEMALES

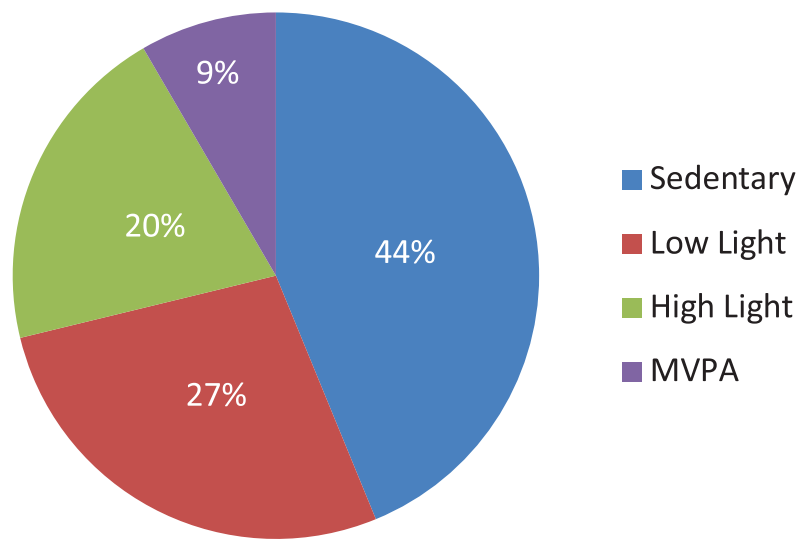

\section{US, FEMALES}

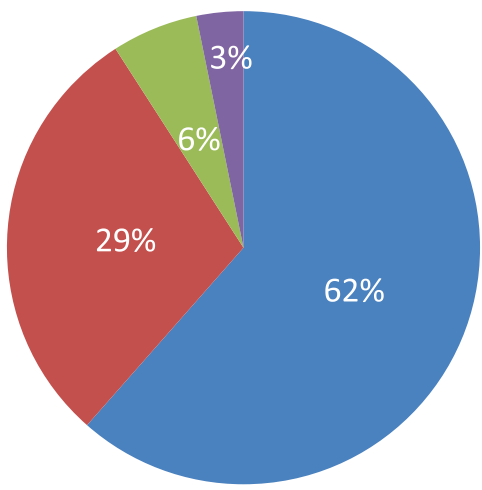

Figure 1 Pie charts for average daily distribution of activity for Malawi and US males and females.

Table 1 Overall and site-specific sample characteristics in Malawi and USA

\begin{tabular}{|c|c|c|c|c|c|c|c|c|c|c|}
\hline & \multicolumn{5}{|l|}{ Malawi } & \multicolumn{5}{|l|}{ US } \\
\hline & \multicolumn{5}{|c|}{ Mean (SD) or \% } & \multicolumn{5}{|c|}{ Mean (SD) or \% } \\
\hline & All agest & $15-19$ & 20-39 & $40-64$ & 65-85 & All ages† & $15-19$ & 20-39 & $40-64$ & $65-85$ \\
\hline Age & $35.1(16.1)$ & $16.7(1.5)$ & $28.9(5.8)$ & $49.1(7.1)$ & $72.0(6.2)$ & $\begin{array}{l}48.6 \\
(19.3)\end{array}$ & $15.6(0.5)$ & $\begin{array}{l}32.2 \\
(4.8)\end{array}$ & $\begin{array}{l}51.0 \\
(6.8)\end{array}$ & $\begin{array}{l}73.7 \\
(5.7)\end{array}$ \\
\hline $\begin{array}{l}\text { Accelerometer } \\
\text { wearing days }\end{array}$ & $13.3(1.5)$ & $12.9(1.8)$ & $13.2(1.6)$ & $13.7(1.1)$ & $13.5(1.6)$ & $6.3(1.4)$ & $5.7(2.0)$ & $6.2(1.5)$ & $6.4(1.4)$ & $\begin{array}{l}6.4 \\
(1.2)\end{array}$ \\
\hline
\end{tabular}

†Samples in Malawi and the USA differed in age $(p<0.001)$ and sex $(p<0.05)$. The Malawi sample had more wearing days $(p<0.001)$ and hours per wearing day $(p<0.001)$ compared with the USA.

PA, high-light PA, moderate PA, vigorous PA, MVPA (sum of moderate and vigorous categories) and total accelerometer counts per day. Sex, age, and accelerometer wear- time covariates. Overall, sample averages and averages for each of the four age groups are provided for both countries. Compared with the USA, Malawi participants 


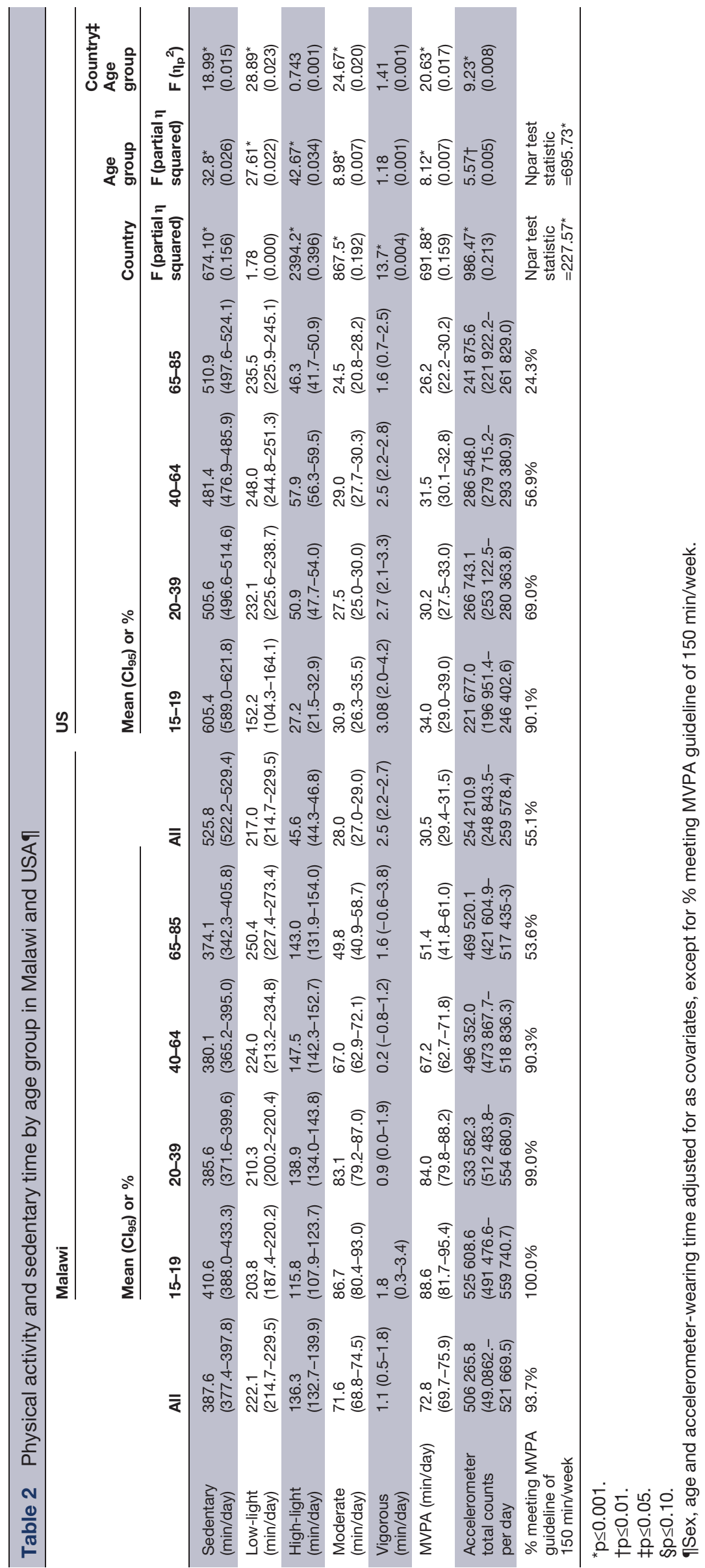


averaged consistently less sedentary time per day and more PA minutes per day in all intensity levels, except for low-light and vigorous PA ( $<3 \mathrm{~min}$ per day, on average, in both countries). Average sedentary time was inversely related to age groups in Malawi and across the three younger age groups in the USA, but sedentary time was higher in the 65-85 age group than the other two adult age groups. Adolescents in the USA and Malawi had more sedentary time than other age groups. Average MVPA minutes per day showed a consistent inverse pattern with age in both countries. MVPA was highest among the adolescents and became progressively lower in older groups. The significant interactions between country-byage group for 4 of $6 \mathrm{PA}$ intensity levels indicated the differences in lower-intensity activity (sedentary and lowlight) were more variable across age groups in the USA, with the greatest differences between the youngest and oldest groups. Higher-intensity activity (moderate PA and MVPA) became progressively lower with each older age group in both the US and Malawi, but the age differences were larger in Malawi. Almost 94\% of Malawi participants met the guideline of $150+\min$ of MVPA per week, whereas only $55 \%$ of US participants did. Meeting the guideline was inversely related to age in both countries.

\section{Physical activity and sedentary time by sex within country}

Table 3 shows the average minutes per day participants spent in each PA-intensity category. In both Malawi and the USA, men spent more time being sedentary than women. Men also had more moderate and total MVPA minutes per day than women in both countries, with the relative differences more in Malawi than in the USA for moderate and vigorous PA. The patterns were mixed for the low-light and high-light PA categories. There was no sex difference for low-light PA in Malawi, but US women had more low-light PA than men. In Malawi, men had significantly less high-light PA than women, but the reverse pattern was found in the US sample.

The distributions of average time spent in different PA-intensity levels varied across the two countries and among men and women. These patterns are illustrated in figure 1. Malawi men and women spent $48 \%$ and $44 \%$ of their accelerometer-wearing time being sedentary, whereas US men and women spent $63 \%$ and $62 \%$ of their time being sedentary. Malawi men and women spent $24 \%$ and $29 \%$ of their time doing high-light and MVPA, in contrast to $10 \%$ and $9 \%$ for US men and women. Men and women in Malawi and the USA spent $27-29 \%$ of their time doing low-light intensity PA.

\section{DISCUSSION}

Overall PA among adults in Malawi was substantially higher than in other population-based samples reported in the literature using hip-worn accelerometers. ${ }^{24}$ The comparison with a US sample from the International Physical Activity and the Environment Network (IPEN) study with a similar age and sex profile, and for which data collection, synthesis, and analysis were carried out nearly identically, put the Malawi data in perspective. The average of $72.8 \mathrm{~min} /$ day of MVPA in Malawi was more than twice the 30.5 min average MVPA of the US sample, and almost twice the average of $37.3 \mathrm{~min}$ among the 14 cities from 10 middle-income and high-income countries in the IPEN Adult Study. ${ }^{24}$ While none of these sites were rural, the IPEN populations that might be perceived as somewhat comparable are the three Latin American LMIC sites. Mean min/day of MVPA in Cuernavaca, Mexico, Curitiba, Brazil and Bogotá, Colombia were 31.2, 31.5, and 37.0, respectively. ${ }^{24}$ Present results are also consistent with those seen in a comparison of rural and urban adults in Cameroon in which rural adults had $107 \mathrm{~min} /$ day of MVPA compared with $62 \mathrm{~min} /$ day of MVPA in urban adults. ${ }^{25}$ While the Cameroon population was a better match with the Malawi population than those noted above, the markedly different measures used to assess PA make direct comparison difficult. The pattern of activity in Malawi shown in figure 1 further differentiated this rural population from previously studied populations in both high-income and middle-income countries. As expected for a rural agricultural population compared with a US urban population, there was much more light activity and correspondingly less sedentary time in the Malawi sample. Adults in Malawi had about threequarters of the daily sedentary time and $40 \%$ more light activity time than the US sample. Essentially all of the difference in light activity was in the 'high-light PA' category. Surprisingly, Malawi adults engaged in even less vigorous $\mathrm{PA}$ than US adults (1.1 vs $2.5 \mathrm{~min} /$ day). The Malawi sample had two and a half times as much moderate PA as the US sample (71.6 vs $28.0 \mathrm{~min} /$ day). The difference was dramatic, illustrated by $93.7 \%$ of the Malawi study population meeting the 150 min per week PA guidelines, compared with $55.1 \%$ in the US sample.

In addition to large differences in overall PA among adults in Malawi compared with the USA, figure 1 demonstrates differences in the distribution of activities across intensity categories. US adults had substantially more sedentary time, and among Malawians, much sedentary time was replaced with both MVPA and high-light PA. Only the proportion of activity time in low-light was similar across all four groups. Given growing evidence of the health benefits of less-than-moderate intensities of $\mathrm{PA},{ }^{26}$ 27 the pattern of PA in Malawi could provide important health benefits if caloric intake was sufficient to support these activity levels.

Despite both expected and unexpected challenges, this study demonstrated it was feasible to collect highquality objective PA data in a very low-resource rural low-income country setting. The familiarity of the field team with carrying out surveys in rural Malawi meant that with appropriate training and support and an existing system of resident enumerators data collection went smoothly. Response rates and wear times were as high or higher than in IPEN countries, and loss of 


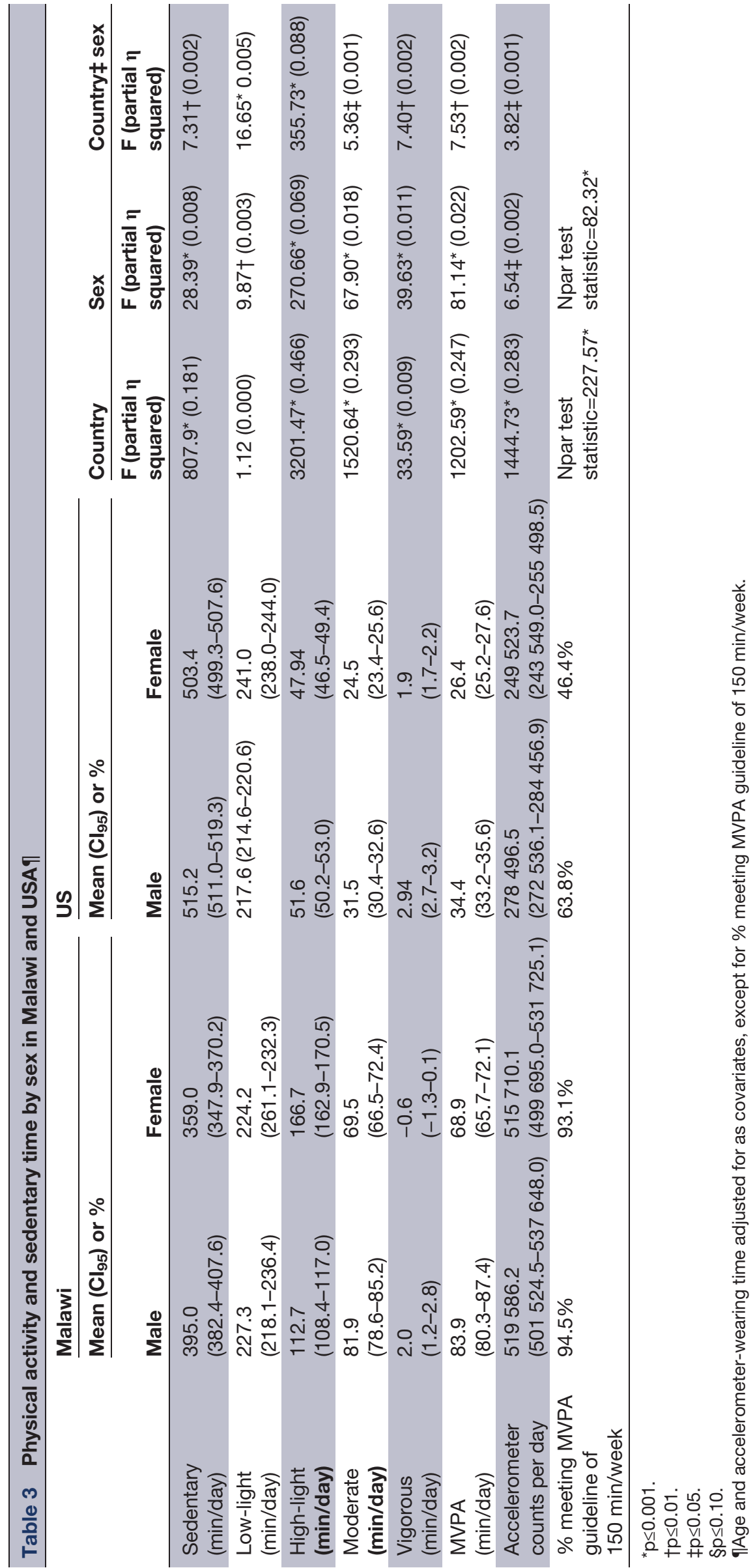


devices was minimal. Compliance was incentivized by providing respondents with small gifts such as soap. Though it would be valuable to increase use of accelerometers in low-income countries, there are several challenges. Funding would likely need to be provided by international sources, as the devices are expensive, and substantial staffing is needed to collect, manage, and process the data. The present approach with local expert data collectors and international coordination to ensure comparable data management and scoring should be considered for future studies.

The results of the present study provide new insights on device-measured PA and sedentary time in rural Malawi, and it would be valuable to evaluate how these finding generalise to other low-income countries and rural populations. The much higher overall PA and lower sedentary time in rural Malawi are consistent with expectations and support the assumption that as rural agricultural populations move to urban areas their PA declines and is replaced by sedentary time. ${ }^{28-30}$ Of course, the present study did not provide a direct test of the hypothesis. Generalisation of this study was also limited because it was carried out in a single country during one season and samples in neither country were nationally representative. Other limitations included differences in the number of days and duration the accelerometers were worn, use of different accelerometer models in the USA and Malawi studies that might not be entirely corrected for with LFE activation, limited ability of hip-worn devices to capture load bearing and upper body activity, higher participation rates in Malawi, and that neither population was representative of the complete rural or urban populations in Malawi or the US However, the coupling of an experienced World Bank survey team with the experienced IPEN coordination team for accelerometer training, data management, quality control, and analysis enhances confidence in data quality. Additional studies of this quality in other low-income and rural settings will be required to further our understanding of PA and sedentary time across the global spectrum of countries. However, it is critical to apply strictly standardised methods of collecting, managing, and scoring accelerometer data, because it is well documented that small variations in methods can dramatically affect outcomes. ${ }^{31-36}$ Previous attempts to compare accelerometer data collected independently in various countries failed due to methodological differences. ${ }^{7}$ Future international PA studies need to use standard protocols and provide equipment, training, ongoing support, and central scoring of data to achieve comparable results. ${ }^{12}$

The current Malawi sample of agricultural workers lacked electricity, motorised vehicles, and most modern technology. Their lifestyle probably resembles those of historical agricultural societies. The data from Malawi provide a rare glimpse into activity patterns in a low-income economy. Impressive differences were observed between adults in Malawi and the USA, with Malawi adults doing about twice as much MVPA per day as the US sample and a sample of adults from 10 middle-income and highincome countries. ${ }^{24}$ If the Malawi pattern of much more MVPA and high-light activity and much less sedentary time was common in recent centuries of human history, it suggests modernisation, motorisation, and technological progress have profoundly changed PA in ways that have been difficult to quantify previously. These Malawi farmers may be an example of 'normal' levels of PA that our biology is adapted for but which our culture has forgotten.

\section{Author affiliations}

${ }^{1}$ Institute for Public Health, University of California, San Diego, La Jolla, California, USA

${ }^{2}$ Family Medicine and Public Health, University of California San Diego, San Diego, California, USA

${ }^{3}$ World Bank Group, Washington, District of Columbia, USA

Acknowledgements The authors wish to thank the full World Bank Rome Office, Malawi Field Team, Wadonda Consult, and University of California San Diego Data Management Team for their contributions to this project. The Malawi Agricultural Labor Experiment 2016/17, inclusive of the PA data collection, was implemented by Wadonda Consult, as a World Bank contractor, with funding from UK Aid, the William and Flora Hewlett Foundation and the World Bank Strategic Research program. The University of California San Diego was also contracted by the World Bank, with funding from the Strategic Research program, to provide technical support to the design and implementation of PA data collection, inclusive of remote field staff training and data quality control. We dedicate this paper to the memory of Professor Ephraim Chirwa.

Contributors JF, TK, JFS, AZ, and MP conceived the idea for this study. The entire author team was involved in study design and implementation. TLC and KLC led the statistical analysis with input and review from all authors. MP, JFS, TLC and KLC drafted the manuscript and all authors contributed to reviewing and editing. All authors approved the final version of the paper and are accountable for all aspects of the work. MP served as the guarantor for the submission. The findings, interpretations and conclusions expressed in this paper are entirely those of the authors. They do not necessarily represent the views of the International Bank for Reconstruction and Development/World Bank and its affiliated organisations, or those of the Executive Directors of the World Bank or the governments they represent.

Funding The 'Measuring Welfare Well - Malawi Physical Activity Tracking Subcomponent' project was funded as contract number 1240680 by the World Bank.

\section{Competing interests None declared.}

Patient consent for publication Patients or the public were involved in the design, or conduct, or reporting, or dissemination plans of our research, providing feedback on survey design and protocol.

Ethics approval The US-based studies providing comparison data were approved by the Institutional Review Boards at the academic institutions that participated in conducting the US research (San Diego State University protocol \#s: 2179, 1294, 2203; University of California San Diego protocol \#s: 121250X, 130615, 121232). The World Bank does not require institutional ethics approval for household surveys, however, informed consent was received from all survey participants.

Provenance and peer review Not commissioned; externally peer reviewed.

Data availability statement Data may be obtained from a third party and are not publicly available. The requests for the anonymised, unit-record data that would be required to replicate the analyses presented in this paper should be directed to Talip Kilic (Senior Economist, World Bank, tkilic@worldbank.org, ORCID: https://orcid.org/ 0000-0002-3642-3123)

Open access This is an open access article distributed in accordance with the Creative Commons Attribution Non Commercial (CC BY-NC 4.0) license, which permits others to distribute, remix, adapt, build upon this work non-commercially, and license their derivative works on different terms, provided the original work is properly cited, appropriate credit is given, any changes made indicated, and the use is non-commercial. See: http://creativecommons.org/licenses/by-nc/4.0/.

\section{ORCID iD}

Michael Pratt http://orcid.org/0000-0002-8939-7715 


\section{REFERENCES}

12018 Physical Activity Guidelines Advisory Committee. 2018 physical activity guidelines advisory committee scientific report. Washington, DC: U.S. Department of Health and Human Services, 2018.

2 World Health Organization. Global action plan for the prevention and control of noncommunicable diseases 2013-2020. Geneva: World Health Organization, 2013.

3 Lee IM, Shiroma EJ, Lobelo F, et al. Effect of physical inactivity on major non-communicable diseases worldwide: an analysis of burden of disease and life expectancy. Lancet 2012;380:219-29.

4 Pratt M, Perez LG, Goenka S, et al. Can population levels of physical activity be increased? Global evidence and experience. Prog Cardiovasc Dis 2015;57:356-67.

5 Kohl HW 3rd, Craig CL, Lambert EV, et al. Lancet physical activity series working group. The pandemic of physical inactivity: global action for public health. Lancet 2012;380:294-305.

6 Guthold R, Stevens GA, Riley LM, et al. Worldwide trends in insufficient physical activity from 2001 to 2016 : a pooled analysis of 358 population-based surveys with 1.9 million participants. Lancet Global Health 2018;6:e1077-e1086.

7 Sallis JF, Bull F, Guthold R, et al. Lancet physical activity series 2 executive committee. progress in physical activity over the olympic quadrennium. Lancet 2016;388:1325-36.

8 Christensen DL, Faurholt-Jepsen D, Boit MK, et al. Cardiorespiratory fitness and physical activity in Luo, Kamba, and Maasai of rural Kenya. Am J Hum Biol 2012;24:723-9.

9 Dugas LR, Bovet P, Forrester TE, et al. Comparisons of intensity-duration patterns of physical activity in the US, Jamaica and 3 African countries. BMC Public Health 2014;14:882.

10 Dugas LR, Kliethermes S, Plange-Rhule J, et al. Accelerometermeasured physical activity is not associated with two-year weight change in African-origin adults from five diverse populations. PeerJ 2017;5:e2902.

11 Assah FK, Ekelund U, Brage S, et al. Urbanization, physical activity, and metabolic health in sub-saharan Africa. Diabetes Care 2011;34:491-6.

12 Kerr J, Sallis JF, Owen N, et al. Advancing science and policy through a coordinated international study of physical activity and built environments: IPEN methods. J Phys Act Health 2013;10:581-601.

13 Cain KL. Accelerometer scoring protocol for the IPEN adult study. 2014. Available http://www.ipenproject.org/methods_accelerometers. html (accessed 27 Jun 2019)

14 Frank LD, Sallis JF, Saelens BE, et al. Development of a walkability index: application to the neighborhood quality of life study. $\mathrm{Br} J$ Sports Med 2010;44:924-33.

15 Sallis JF, Conway TL, Cain KL, et al. Neighborhood built environment and socioeconomic status in relation to physical activity, sedentary behavior, and weight status of adolescents. Prev Med 2018;110:47-54.

16 King AC, Sallis JF, Frank LD, et al. Aging in neighborhoods differing in walkability and income: associations with physical activity and obesity in older adults. Soc Sci Med 2011;73:1525-33.

17 Sallis JF, Saelens BE, Frank LD, et al. Neighborhood built environment and income: examining multiple health outcomes. Soc Sci Med 2009;68:1285-93.

18 Sirard JR, Slater ME. Compliance with wearing physical activity accelerometers in high school students. J Phys Act Health 2009;6: S148-155.
19 Cain KL, Conway TL, Adams MA, et al. Comparison of older and newer generations of ActiGraph accelerometers with the normal filter and the low frequency extension. Int J Behav Nutr Phys Act 2013;10:51.

20 Ried-Larsen M, Brønd JC, Brage S, et al. Mechanical and free living comparisons of four generations of the Actigraph activity monitor. Int J Behav Nutr Phys Act 2012;9:113.

21 Freedson PS, Melanson E, Sirard J. Calibration of the computer science and applications, Inc. accelerometer. Med Sci Sports Exerc 1998;30:777-81.

22 Cain KL, Geremia CM. Accelerometer data collection and scoring manual for adult \& senior studies. San Diego, CA: San Diego State University, 2012

23 Matthews CE, Chen KY, Freedson PS, et al. Amount of time spent in sedentary behaviors in the United States, 2003-2004. Am J Epidemiol 2008;167:875-81.

24 Sallis JF, Cerin E, Conway TL, et al. Urban environments in 14 cities worldwide are related to physical activity. Lancet 2016;387:2207-17.

25 Assah F, Mbanya JC, Ekelund U, et al. Patterns and correlates of objectively measured free-living physical activity in adults in rural and urban Cameroon. J Epidemiol Community Health 2015;69:700-7.

26 LaCroix AZ, Bellettiere J, Rillamas-Sun E, et al. Association of light physical activity measured by accelerometry and incidence of coronary heart disease and cardiovascular disease in older women. JAMA Network Open 2019;2:e190419-e190419.

27 Ekelund U, Tarp J, Steene-Johannessen J, et al. Dose-response associations between accelerometry measured physical activity and sedentary time and all cause mortality: systematic review and harmonised meta-analysis. BMJ 2019;366:14570.

28 Sobngwi E, Mbanya JC, Unwin NC, et al. Exposure over the life course to an urban environment and its relation with obesity, diabetes, and hypertension in rural and urban Cameroon. Int $J$ Epidemiol 2004;33:769-76.

29 Ntandou G, Delisle H, Agueh V, et al. Physical activity and socioeconomic status explain rural-urban differences in obesity: a cross-sectional study in Benin (West Africa). Ecol Food Nutr 2008;47:313-37.

30 Sobngwi E, Mbanya JN, Unwin NC, et al. Physical activity and its relationship with obesity, hypertension and diabetes in urban and rural Cameroon. Int J Obes 2002;26:1009.

31 Cain KL, Sallis JF, Conway TL, et al. Using accelerometers in youth physical activity studies: a review of methods. J Phys Act Health 2013;10:437-50.

32 Orme M, Wijndaele K, Sharp SJ, et al. Combined influence of epoch length, cut-point and bout duration on accelerometry-derived physical activity. Int J Behav Nutr Phys Act 2014;11:34. Available http://www. ijbnpa.org/content/11/1/34

33 Mota J, Valente M, Aires L, et al. Accelerometer cut points and youth physical activity prevalence. Eur Phys EducRev 2007;13:287-99.

34 Evenson K, Terry X. Assessment of differing definitions of accelerometer nonwear time. Res Q Exerc Sport 2009;80:355-62.

35 Cain KL, Bonilla E, Conway TL, et al. Defining accelerometer nonwear time to maximize detection of sedentary time in youth. Pediatr Exerc Sci 2017;30:288-95.

36 Corder K, Ekelund U, Steele RM, et al. Assessment of physical activity in youth. J Appl Physiol 2008;105:977-87. 